\title{
The association between renal tumour scoring systems and ischemia time during open partial nephrectomy
}

\author{
Luke T. Lavallée, MD; ${ }^{*}$ Darren Desantis, MD; Fadi Kamal, MD, FRCSC; ${ }^{*}$ Brian Blew, MD, FRCSC; ${ }^{*}$ James \\ Watterson, MD, FRCSC; ${ }^{*}$ Ranjeeta Mallick, PhD; ${ }^{\dagger}$ Dean Fergusson, PhD; ${ }^{\dagger}$ Christopher Morash, MD, FRCSC; ${ }^{*}$ \\ Ilias Cagiannos, MD, FRCSC,; Rodney H. Breau, MD, FRCSC*
}

*Division of Urology, Department of Surgery, The Ottawa Hospital and University of Ottawa, Ottawa, ON; †Ottawa Hospital Research Institute, Ottawa ON

Cite as: Can Urol Assoc J 2013;7:E207-14. http://dx.doi.org/10.5489/cuaj.11202. Epub 2012 May 15.

\section{Abstract}

Objective: To evaluate the association between renal tumour scoring systems and open partial nephrectomy ischemia time.

Methods: A historical cohort of open partial nephrectomy patients at The Ottawa Hospital between 2002 and 2009 was reviewed. Preoperative patient characteristics (age, gender, preoperative renal function, diabetes, hypertension, smoking history, heart disease) and ischemia time were abstracted from medical records. Preoperative computed tomography $(\mathrm{CT})$ images were reviewed and tumours were characterized using three scoring systems: (1) R.E.N.A.L. nephrometry score (radius, exophytic/endophytic properties, nearness of tumour to the collecting system or sinus in millimetres, anterior/posterior, location relative to polar lines); (2) preoperative aspects and dimensions used for anatomic (PADUA) classification; and (3) Centrality index (C index). Patients without preoperative CT and patients treated with laparoscopic partial nephrectomy were excluded.

Results: During the study period, 78 patients met the inclusion criteria. Median R.E.N.A.L. score was 7 (interquartile range [IQR] 5-8), median PADUA score was 8 (IQR 7-10), and mean C index was 3.9 (standard deviation [SD] 2.1). Mean ischemia time was 23.4 (SD 10.8) minutes. Five individual tumour characteristics (diameter, nearness to collecting system, anterior/posterior location, medial/lateral location, and collecting system involvement) were strongly associated with ischemia time $(p<0.05)$. Increased R.E.N.A.L. score (1.5 minutes per unit $95 \% \mathrm{Cl} 0.08,2.9, p=0.04)$ and PADUA score $(2.0$ minutes per unit $95 \% \mathrm{Cl} 0.5,3.5, p=0.009)$ were significantly associated with ischemia time. An increasing $C$ index score was also associated with ischemia time $(-1.1$ minutes per unit $95 \% \mathrm{Cl}-2.2,0.04, p=0.06)$, but the association was not statistically significant.

Conclusion: Renal tumour characteristics are associated with ischemia time. The proposed scoring systems are useful descriptors of surgical complexity and should be used when describing partial nephrectomy patients. Prospective evaluation and refinement of scoring systems are required to create an optimized model prior to widespread application.

\section{Introduction}

The decision to perform radical versus partial nephrectomy for renal tumours is currently based on the subjective assessment of feasibility and patient preferences. Renal tumour scoring systems are designed to characterize tumours, facilitate cohort comparisons and allow for the prediction of surgical outcomes. Three scoring systems have been proposed and require validation: (1) R.E.N.A.L. nephrometry score (radius, exophytic/endophytic properties, nearness of tumour to the collecting system or sinus in millimeters, anterior/ posterior, location relative to polar lines); (2) preoperative aspects and dimensions used for anatomic (PADUA) classification; and (3) Centrality index (C index). ${ }^{1-3}$ The R.E.N.A.L. and PADUA systems categorize tumour characteristics and provide an overall score with a high score associated with more complex features. The $\mathrm{C}$ index method derives one number that reflects tumour size and distance from the centre of the kidney with a lower score representing tumours that are larger and closer to the kidney centre.

Scoring systems are helpful if they are able to predict outcomes, including technical difficulties of partial nephrectomy, risk of complications (i.e., bleeding, urine leak, need for conversion to radical nephrectomy) and functional/ oncologic outcomes (i.e., change in creatinine, risk of recurrence). The original publications and a small number of additional studies have examined some of these outcomes. ${ }^{1-9}$ To the best of our knowledge, this is the first study applying all three scoring systems to a cohort of patients treated with open partial nephrectomy to determine which system is most predictive of ischemia time.

We postulated that the individual components and overall scores of the R.E.N.A.L. (Table 1), PADUA and the C index systems are associated with surgical complexity/difficulty. Therefore, if tumour scoring systems predict complexity, we would expect higher R.E.N.A.L. and PADUA scores, and lower $\mathrm{C}$ index scores, to be associated with longer ischemia 


\begin{tabular}{|c|c|c|c|}
\hline & $1 \mathrm{pt}$ & 2 pts & 3 pts \\
\hline Radius (maximum diameter in $\mathrm{cm}$ ) & $\leq 4$ & $5-6$ & $\geq 7$ \\
\hline Exophytic/endophytic properties & $\geq 50 \%$ & $<50 \%$ & Entirely endophytic \\
\hline $\begin{array}{l}\text { Nearness of the tumour to the } \\
\text { collecting system or sinus }(\mathrm{mm})\end{array}$ & $\geq 7$ & $5-6$ & $\leq 4$ \\
\hline Anterior/posterior & \multicolumn{3}{|c|}{ No points given. Mass assigned a descriptor of $a, p$ or $x$. } \\
\hline Location relative to the polar lines* & $\begin{array}{l}\text { Entirely above the upper or } \\
\text { below the lower polar line }\end{array}$ & Lesion crosses polar line & $\begin{array}{c}>50 \% \text { of mass is across polar line (a) or mass } \\
\text { crosses the axial renal midline (b) or mass is } \\
\text { entirely between the polar line (c) }\end{array}$ \\
\hline
\end{tabular}

time during surgery. The purpose of this study was to apply each scoring system to a cohort of patients who received open partial nephrectomy at our institution and to evaluate the strength of the association between tumour scores and ischemia time.

\section{Methods}

Institutional ethics review board approval was obtained to review a historical cohort of patients who underwent open partial nephrectomy for renal tumours between 2002 and 2009 by two surgeons at one tertiary care institution (The Ottawa Hospital, Ottawa, Ontario). Patients without preoperative computed tomography (CT) and patients treated with laparoscopic partial nephrectomy were excluded. Abstracted patient characteristics, including age, gender, pre-/postoperative serum creatinine, diabetes, hypertension, smoking history and heart disease, were recorded. Ischemia time was prospectively documented during each procedure. All preoperative CT scans of the abdomen and pelvis were reviewed by two independent physicians (LL, DD). Tumour characteristics and scores were recorded by applying the criteria outlined by each renal tumour scoring systems (R.E.N.A.L., PADUA, C index). ${ }^{1-3}$

\section{Predictor variables and scoring systems}

The R.E.N.A.L. and PADUA systems are composite scores that describe renal masses in a quantifiable way. They incorporate tumour diameter, tumour location within the kidney (e.g., anterior/posterior, medial/lateral, polar/non-polar) and tumour association with other structures (e.g., renal sinus or collecting system). Although the R.E.N.A.L. and PADUA systems have many similarities, they differ in their incorporation and definition of several variables. Although both systems propose that the polar location is an important variable, they stratify tumour polarity differently. The $\mathrm{C}$ index score is derived from a series of measurements made on cross-sectional imaging. It describes tumour size and position relative to the centre of the kidney.

\section{Outcome variables}

Each scoring system was evaluated for their associations with ischemia time (primary outcome) and perioperative change in serum creatinine concentration (secondary outcome). Ischemia time was extracted from the operative record and refers to the duration of time required for tumour excision and renal reconstruction; it was used as an indicator for surgical complexity. Change in renal function was assessed using standardized laboratory assays by measuring the difference in serum creatinine concentration preoperatively compared to 3 months postoperatively.

\section{Statistical analysis}

Inter-rater reliability was assessed to quantify the agreement between reviewers for each tumour score component using Pearson and Kappa analytic techniques, as appropriate. Univariate linear regression analysis determined the association between scoring systems, individual tumour characteristics, and clinical characteristics with ischemia time and perioperative change in creatinine. A multivariable linear regression model was created to evaluate the independent associations between each tumour component and ischemia time or perioperative change in creatinine. Components that are not incorporated into the overall numerical score, such as the anterior/posterior location (R.E.N.A.L., PADUA) and hilar association (R.E.N.A.L.), were evaluated independently, but were not included in the multivariable analysis. Tumour predictor variables for R.E.N.A.L. and PADUA were treated categorically. C index characteristics/scores and overall R.E.N.A.L. and PADUA scores were treated as continuous variables.

To compare tumour scoring models, the coefficient of determination $\left(\mathrm{r}^{2}\right)$ was calculated. The $\mathrm{r}^{2}$ was determined for multivariable models that incorporated the overall score (R.E.N.A.L., PADUA or C index) and clinical information (age, sex, preoperative creatinine, diabetes, smoking history, hypertension and heart disease). The $r^{2}$ value indicates what proportion of the variability in outcome (ischemia time or 
change in creatinine) is explained by the scoring models and clinical information combined.

\section{Results}

A total of 110 open partial nephrectomies were performed by two surgeons at The Ottawa Hospital from 2002 to 2009. After excluding patients without available preoperative CT imaging ( $\mathrm{n}=32), 78$ were reviewed. Of the 78, 6 did not have documented ischemia times and were excluded from analyses evaluating predictors of ischemia time. We tallied their clinicopathological characteristics (Table 2).

\section{Tumour characteristics and inter-rater agreement}

Inter-rater agreement was good for all tumour characteristics and scoring systems. $\mathrm{C}$ index had a higher degree of agreement compared to R.E.N.A.L. or PADUA (Table 3).

\section{Ischemia time}

For the primary analysis, the associations between scoring systems and ischemia time were evaluated (Table 4). Both R.E.N.A.L. and PADUA scores were significantly associated with ischemia time. For every increase in R.E.N.A.L. score of 1 , an increase in average ischemia time of 1.5 minutes was observed $(95 \% \mathrm{Cl}$ [confidence interval] $0.08,2.9, p=0.04)$. For PADUA, the magnitude of association was larger. An increase in an overall PADUA score of 1 was associated with an additional 2.0 minutes of ischemia time $(95 \% \mathrm{Cl}$ $0.5,3.5, p=0.009$ ), on average. The overall $\mathrm{C}$ index score was also associated with ischemia time, for every increase in $\mathrm{C}$ index unit of 1 , a decrease of 1.1 minutes $(95 \% \mathrm{Cl}$ $-2.2,0.04, p=0.06$ ) was observed; however this association was not statistically significant in this series. The $r^{2}$ value for each scoring system model were R.E.N.A.L. (13.5\%), PADUA (17.1\%) and C index (13.4\%), indicating that none of the models explained a high proportion of ischemia time variability.

On univariate analysis, no association was found between any of the clinical characteristics and ischemia time (Table 2). Strong associations were observed between the following predictor variables and ischemia time: diameter (C index), nearness (R.E.N.A.L.), anterior/posterior location (R.E.N.A.L., PADUA), collecting system involvement (PADUA), and medial/lateral location (PADUA) (Table 4). On average, for each additional centimetre of tumour diameter, an additional 1.9 minutes $(95 \% \mathrm{Cl} 0.2,3.6, p=0.04)$ of ischemia time was required. If a tumour was $\leq 4 \mathrm{~mm}$ from the renal sinus/ collecting system, it was associated with 6.1 minutes more ischemia time $(95 \% \mathrm{Cl} 1.0,11.3, p=0.02)$ compared to a tumour $\geq 7 \mathrm{~mm}$ from the sinus/collecting system. Tumours associated with the collecting system according to the PADUA system were associated with 5.4 minutes more ischemia time than those that were not $(95 \% \mathrm{Cl}-10.7,0.0, p=0.05)$. On average, medially located tumours were associated with 7.9 minutes $(95 \% \mathrm{Cl} 3.1,12.7, p=0.002)$ more ischemia time than lateral tumours. Tumours in posterior locations

\begin{tabular}{|c|c|c|c|c|c|}
\hline \multirow{2}{*}{ Demographics } & \multirow{2}{*}{ n (SD or \%) } & \multicolumn{2}{|c|}{ Ischemia time } & \multicolumn{2}{|c|}{ Change in creatinine } \\
\hline & & Coefficient 95\% Cl & $p$ value & Coefficient 95\%Cl & $p$ value \\
\hline Age (years) & $61.0(13.5)$ & $0.1(-0.06,0.3)$ & 0.2 & $0.4(0.05,0.8)$ & 0.03 \\
\hline Sex & & $0.06(-5.4,5.5)$ & 1.0 & $6.6(-5.6,18.8)$ & 0.3 \\
\hline Male & $55(70.5 \%)$ & & & & \\
\hline Female & $23(29.5 \%)$ & & & & \\
\hline Preoperative creatinine & $100.6(40.3)$ & $0.04(-0.02,0.1)$ & 0.2 & $0.07(-0.07,0.2)$ & 0.3 \\
\hline Diabetes & $13(16.7 \%)$ & $1.3(-8.9,5.4)$ & 0.7 & $-5.9(-20.9,9.1)$ & 0.3 \\
\hline Hypertension & $39(50.0 \%)$ & $-4.1(-9.0,0.8)$ & 0.1 & $-4.9(-16.1,6.2)$ & 0.4 \\
\hline Heart disease & $25(32.1 \%)$ & $2.4(-2.9,7.6)$ & 0.4 & $-6.4(-18.3,5.6)$ & 0.3 \\
\hline Smoking history & $48(61.5 \%)$ & $-1.7(-6.8,3.4)$ & 0.5 & $-7.3(-18.7,4.1)$ & 0.2 \\
\hline \multicolumn{6}{|l|}{ Kidney } \\
\hline Right (\%) & $36(46.2 \%)$ & & & & \\
\hline Left (\%) & $42(53.9 \%)$ & & & & \\
\hline Estimated blood loss (cc) & $351(617)$ & & & & \\
\hline Ischemia time (minutes) & $23.4(10.8)$ & & & & \\
\hline$\%$ Positive margin & $3(3.9 \%)$ & & & & \\
\hline \multicolumn{6}{|l|}{ Tumour pathology } \\
\hline Malignant (\%) & $60(76.9 \%)$ & & & & \\
\hline Benign (\%) & $18(23.1 \%)$ & & & & \\
\hline Tumour diameter $(\mathrm{cm})$ & $2.7(1.6)$ & & & & \\
\hline
\end{tabular}




\begin{tabular}{|c|c|c|}
\hline Variable & Value & $\begin{array}{l}\text { Inter-rate } \\
\text { agreement } \\
\text { coefficient }\end{array}$ \\
\hline \multicolumn{3}{|l|}{ NEPHROMETRY SCORE } \\
\hline Diameter (cm) & & $0.9(0.7,1.0)$ \\
\hline$\leq 4$ & $66(84.6 \%)$ & \\
\hline $5-6$ & $12(15.4 \%)$ & \\
\hline$\geq 7$ & $0(0 \%)$ & \\
\hline Exophytic/endophytic & & $0.5(p<0.0001)$ \\
\hline$\geq 50 \%$ exophytic & $28(35.9 \%)$ & \\
\hline$<50 \%$ exophytic & $48(61.5 \%)$ & \\
\hline Entirely endophytic & $2(2.6 \%)$ & \\
\hline Nearness (mm) & & $0.7(0.6,0.8)$ \\
\hline$\geq 7$ & $37(47.4 \%)$ & \\
\hline $5-6$ & $9(11.5 \%)$ & \\
\hline$\leq 4$ & $32(41.0 \%)$ & \\
\hline $\begin{array}{l}\text { Polar location (relative to } \\
\text { polar lines) }\end{array}$ & & $0.8(0.7,0.9)$ \\
\hline Entirely above polar line & $34(43.6 \%)$ & \\
\hline Crosses polar line & $20(25.6 \%)$ & \\
\hline $\begin{array}{l}>50 \% \text { across polar line or } \\
\text { crosses axial midline or } \\
\text { entirely between polar lines }\end{array}$ & $24(30.8 \%)$ & \\
\hline Anterior posterior & & $0.6(0.5,0.7)$ \\
\hline Anterior & $33(42.3 \%)$ & \\
\hline Posterior & $31(39.7 \%)$ & \\
\hline Could not assess & $14(17.9 \%)$ & \\
\hline Overall & & $0.6(0.5,0.7)$ \\
\hline Median & 7 (IOR 5-8) & \\
\hline 4 & $10(12.8 \%)$ & \\
\hline 5 & $11(14.1 \%)$ & \\
\hline 6 & $16(20.5 \%)$ & \\
\hline 7 & $18(23.1 \%)$ & \\
\hline 8 & $9(11.5 \%)$ & \\
\hline 9 & $12(15.4 \%)$ & \\
\hline 10 & $2(2.6 \%)$ & \\
\hline Risk group & & $0.5(0.4,0.7)$ \\
\hline Low & $37(47.3 \%)$ & \\
\hline Moderate & $39(50.0 \%)$ & \\
\hline High & $2(2.6 \%)$ & \\
\hline
\end{tabular}

were associated with an average of 6.1 minutes $(95 \% \mathrm{Cl} 0.8$, $11.4, p=0.03)$ more ischemia time than anterior tumours. Other predictor variables were associated with ischemia time, however these were not statistically significant (Table 4). We also tallied the independent associations of score components with ischemia time (Table 5). C index diameter and PADUA medial/lateral location were the only components independently associated with ischemia time in a statistically significant fashion when controlling for the other components of the respective scoring systems $(p<0.05)$.

\begin{tabular}{|c|c|c|}
\hline \multicolumn{3}{|l|}{ Table 3. cont'd } \\
\hline Variable & Value & $\begin{array}{l}\text { Inter-rate } \\
\text { agreement } \\
\text { coefficient }\end{array}$ \\
\hline \multicolumn{3}{|l|}{ PADUA SCORE } \\
\hline Diameter (cm) & & $0.9(0.7,1.0)$ \\
\hline$\leq 4$ & $66(84.6 \%)$ & \\
\hline $5-6$ & $12(15.4 \%)$ & \\
\hline$\geq 7$ & $0(0 \%)$ & \\
\hline Polar location & & $0.8(0.6,0.9)$ \\
\hline Superior/inferior & $32(41.0 \%)$ & \\
\hline Middle & $46(59.0 \%)$ & \\
\hline Exophytic & & $0.5(p<0.0001)$ \\
\hline$\geq 50 \%$ exophytic & $28(35.9 \%)$ & \\
\hline$<50 \%$ exophytic & $48(61.5 \%)$ & \\
\hline Entirely endophytic & $2(2.6 \%)$ & \\
\hline Renal rim & & $0.8(0.7,0.9)$ \\
\hline Lateral & $47(60.2 \%)$ & \\
\hline Medial & $31(39.7 \%)$ & \\
\hline Renal sinus & & $0.7(0.6,0.9)$ \\
\hline Not involved & $49(62.8 \%)$ & \\
\hline Involved & $29(37.2 \%)$ & \\
\hline Urinary collecting system & & $0.5(0.3,0.7)$ \\
\hline Not involved & $55(70.5 \%)$ & \\
\hline Involved & $23(29.5 \%)$ & \\
\hline Face & & $0.6(0.5,0.7)$ \\
\hline Anterior & $33(42.3 \%)$ & \\
\hline Posterior & $31(39.7 \%)$ & \\
\hline Could not access & $14(17.9 \%)$ & \\
\hline \multicolumn{3}{|l|}{ Overall score } \\
\hline Median & 8 (IQR 7-10) & \\
\hline 6 & $7(9.0 \%)$ & \\
\hline 7 & $15(19.2 \%)$ & \\
\hline 8 & $26(33.3 \%)$ & \\
\hline 9 & $8(10.3 \%)$ & \\
\hline 10 & $9(11.5 \%)$ & \\
\hline 11 & $12(15.4 \%)$ & \\
\hline 12 & $1(1.3 \%)$ & \\
\hline 13 & $0(0 \%)$ & \\
\hline \multicolumn{3}{|l|}{ C Index } \\
\hline Horizontal distance $-\mathrm{x}(\mathrm{cm})$ & $2.7(1.0)$ & $0.9(p<0.0001)$ \\
\hline Vertical distance - y (cm) & $2.8(1.8)$ & $0.9(p<0.0001)$ \\
\hline Diameter & $2.7(1.4)$ & $0.9(p<0.0001)$ \\
\hline C & $4.2(1.2)$ & $0.9(p<0.0001)$ \\
\hline C index & $3.9(2.1)$ & $0.9(p<0.0001)$ \\
\hline
\end{tabular}

\section{Change in serum creatinine}

None of the overall system scores were statistically associated with perioperative change in creatinine (Table 4). The proportion of perioperative change in creatinine explained by each scoring system was low: R.E.N.A.L. $\left(r^{2}=7.9 \%\right)$, PADUA $\left(r^{2}=8.0 \%\right)$ and $C$ index $\left(r^{2}=7.9 \%\right)$. Patient age, 


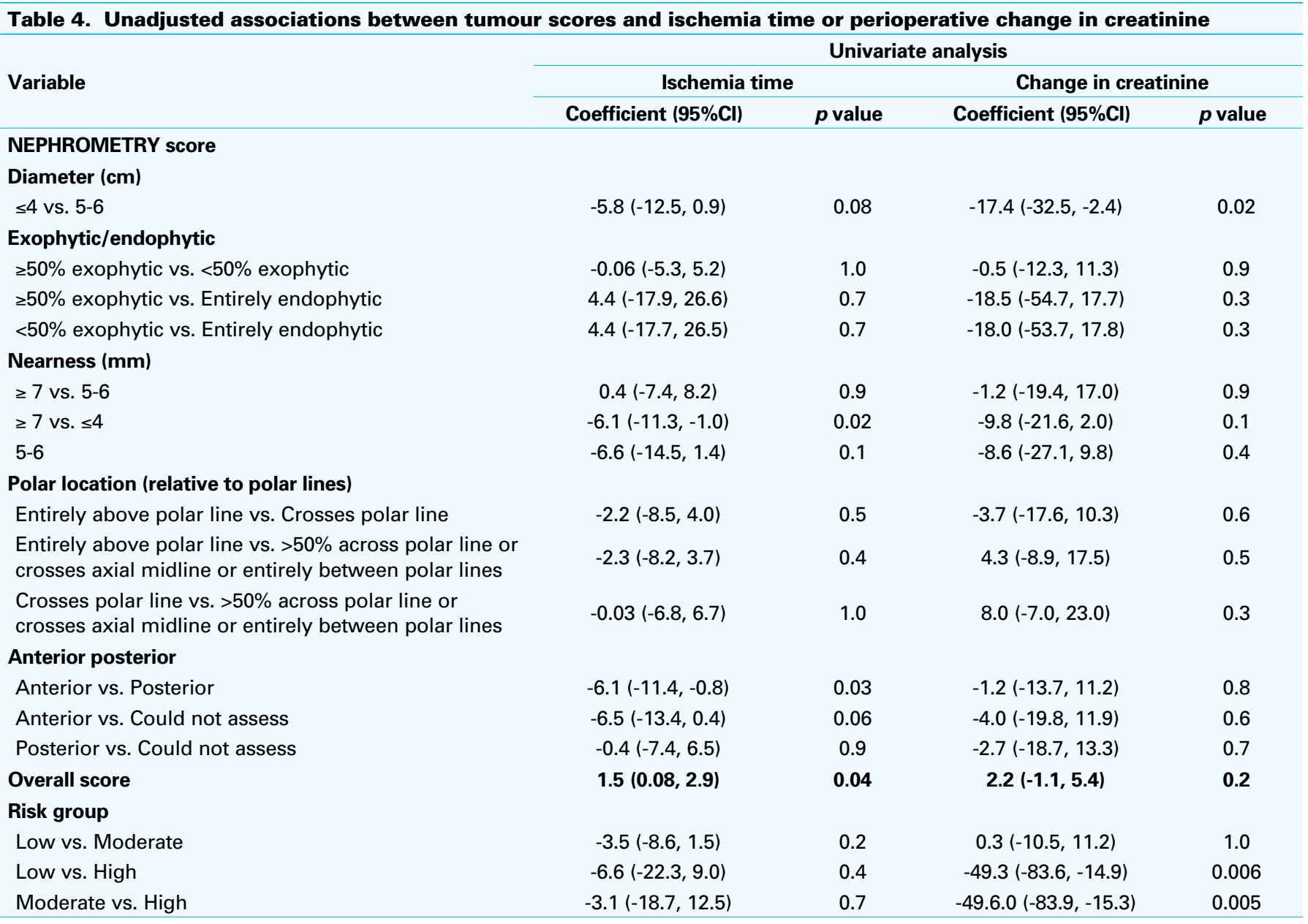

categorical tumour diameter (R.E.N.A.L. and PADUA) and risk group (R.E.N.A.L.) were the only predictor variables that were associated with perioperative change in creatinine in univariate analysis in a statistically significant manner (Table 4). In multivariable analysis, adjusting for the other components of each respective scoring system, only the C index defined diameter was significantly associated with perioperative change in creatinine $(p<0.05)$ (Table 5).

\section{Discussion}

The objective of partial nephrectomy is to achieve equivalent cancer control to radical nephrectomy while preserving renal function. ${ }^{10-13}$ Partial nephrectomy is the more complex surgical procedure and is associated with increased risk of perioperative complications compared to radical nephrectomy. ${ }^{14,15}$ Therefore, patients are offered partial nephrectomy if the perceived benefits outweigh the subjective risk. Until recently, no objective criteria were used to define the complexity of a renal tumour. Thus, reported benefits and risks of partial nephrectomy for individual patients could be over or underestimations of the truth. To address this deficiency in objective criteria, three renal tumour scoring systems have been proposed: R.E.N.A.L., PADUA and C index.

Quantifying case complexity is not easy. We hypothesized that ischemia time is a good representative of case complexity since less complex cases should require less ischemia time to perform the tumour removal and kidney reconstruction compared to more complex cases. We observed that overall PADUA and R.E.N.A.L. scores were associated with ischemia time. As the scores increased (indicating more complex tumours), the ischemia time increased. For every increase in an overall PADUA score of 1 , the ischemia time increased by an average of 2.0 minutes $(95 \% \mathrm{Cl} 0.5,3.5$, $p=0.009$. For every increase in a R.E.N.A.L. score of 1 , the ischemia time increased by an average of 1.5 minutes $(95 \% \mathrm{Cl} 0.08,2.9, p=0.04)$. For every increase in $\mathrm{C}$ index of 1 (indicating either a smaller tumour and/or a tumour more distant from the centre of the kidney), the ischemia time decreased by an average of 1.1 minutes $(95 \% \mathrm{Cl}-2.2$, $0.04, p=0.06)$. However, the $r^{2}$ value revealed that although the scoring systems were predictive of ischemia time, they account for only a small proportion of the ischemia time variability. 


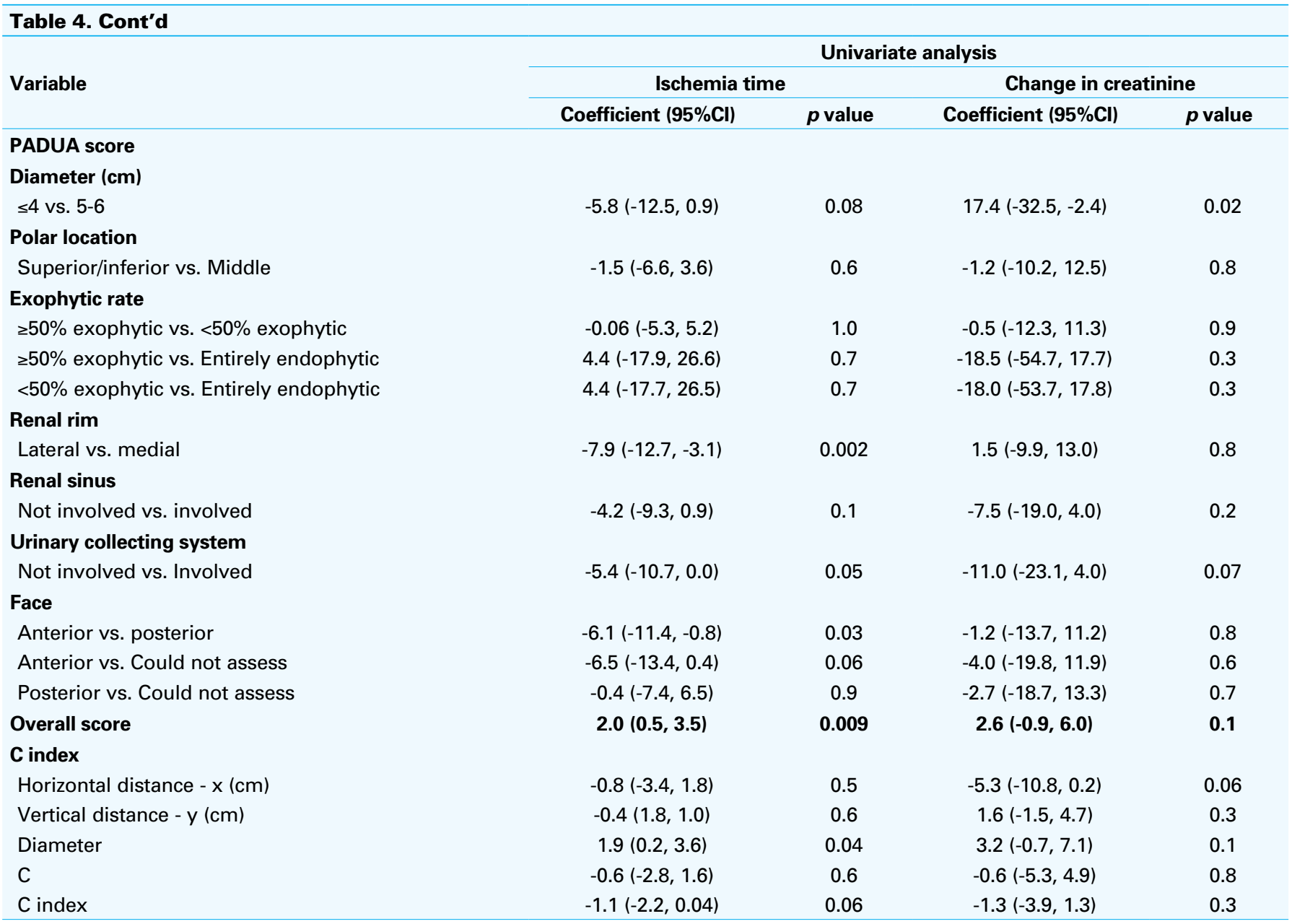

The results of this study are consistent with previously reported series of open, laparoscopic and robotic partial nephrectomies in which PADUA, R.E.N.A.L. or C Index scores were applied. ${ }^{3,6-9}$ To the best of our knowledge, this is the first study comparing all three scoring systems with ischemia time in patients undergoing open surgery. Other authors have stratified R.E.N.A.L. and PADUA scores into risk groups predicting ischemia time; our data revealed similar associations, however, these were not statistically significant. ${ }^{7,8}$

Systems to describe renal tumours are needed and the three proposed scoring systems appear to improve the current standard. The proposed models quantify tumour characteristics and have some value in predicting surgical complexity. However, these data would suggest that further validation and refinement are needed prior to widespread acceptance. $^{4-7}$ The R.E.N.A.L. score was derived based on what the authors felt were important tumour characteristics rather than with statistical validation against an important outcome. ${ }^{2}$ The PADUA classification and $\mathrm{C}$ index methods compared their components to clinical outcomes in the initial publications, but further study is required to determine if these components are optimally defined and weighted. ${ }^{1,3}$ For example, although tumour location and collecting system involvement are associated with ischemia time, perhaps one of these components is significantly more important than the other. This needs to be examined and reflected before a definitive scoring system is universally applied.

We found each of the scoring systems easy to use, as inter-rater agreement was good. $\mathrm{C}$ index had the least interrater variation, likely due to fewer individual components and measurements. Clearly, renal tumour scoring systems should be simple to apply and should eliminate extraneous components that are not consistently predictive of clinically important outcomes.

Several limitations should be considered when interpreting these data. This study does not evaluate the ability of the tumour scoring systems to evaluate the largest and most complicated tumours, since the cohort did not contain tumours $>7 \mathrm{~cm}$ in size. Therefore, the utility of the scoring systems for the most complex tumours is yet to be defined. In addition, a larger sample size would be required to detect 
Table 5. Adjusted associations between scoring system components and ischemia time or peri-operative change in creatinine.

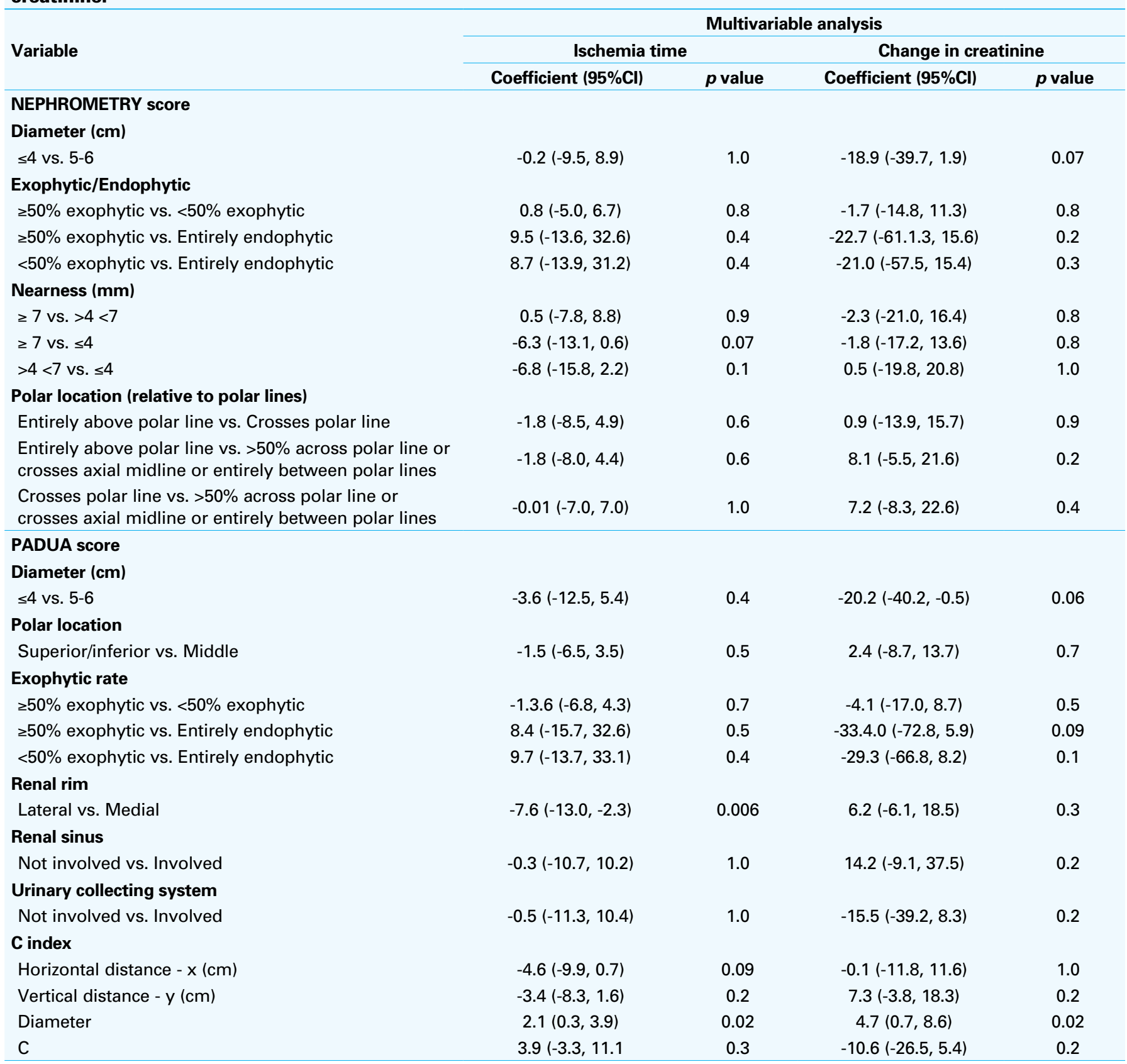

small differences in tumour scoring systems and individual component associations. Serum creatinine is a suboptimal method of measuring perioperative change in renal function; future studies using more accurate measurements of renal function are required prior to making conclusions regarding the association of scoring systems and perioperative change in renal function.

Ideally, to develop the most efficient predictive model, various tumour characteristics would be independently evaluated to determine associations with important clinical outcomes and the resultant model would be prospectively validated in surgical patients. In current scoring systems, the choice of components, stratification methods (categorical vs. continuous) and the method of weighting require further refinement. For example, the point designation for the component nearness (R.E.N.A.L.) stratifies tumours from $\geq 7,5-6$ and $\leq 4 \mathrm{~mm}$ from the collecting system/sinus for simplicity, not because these categories are associated with different outcomes. Also, if one predictor variable (e.g., tumour diameter) is more highly associated with outcomes than other 
Lavallée et al.

components, it may warrant increased influence over the overall scoring system than less important components. This series found that the anterior/posterior location (R.E.N.A.L., PADUA) was significantly associated with ischemia time, yet neither system incorporates this variable into the numerical score, only a qualitative descriptor is used, which renders the clinical application more cumbersome. Finally, the purpose of ischemia time in this study was to determine the complexity of surgical resection. While renal ischemia time is likely the easiest outcome to measure and analyze, the clinical importance of small changes in ischemia times (adjusting for tumour characteristics) is yet to be defined.

\section{Conclusion}

Both R.E.N.A.L. and PADUA scores are significantly associated with ischemia time. Some individual renal tumour characteristics are also associated with ischemia time. The proposed scoring systems are useful descriptors of tumour complexity, but they require further refinement prior to widespread use. The degree of variability of ischemia time that is explained by current scoring systems is low; therefore, we need further exploration of models that may be more predictive of ischemia time.

Competing interests: None declared.

This paper has been peer-reviewed.

\section{References}

1. Ficarra V, Novara G, Secco S, et al. Preoperative aspects and dimensions used for an anatomical (PADUA) classification of renal tumours in patients who are candidates for nephron-sparing surgery. Eur Urol 2009;56:786-93. http://dx.doi.org/10.1016/i.eururo.2009.07.040
2. Kutikov A, Uzzo RG. The R.E.N.A.L. nephrometry score: a comprehensive standardized system for quantitating renal tumor size, location and depth. J Urol 2009;182:844-53. http://dx.doi.org/10.1016/i. juro.2009.05.035

3. Simmons MN, Ching CB, Samplaski MK, et al. Kidney tumor location measurement using the C index method. J Urol 2010;183:1708-13. http://dx.doi.org/10.1016/i.juro.2010.01.005

4. Bruner $B$, Breau RH, Lohse $C M$, et al. Renal nephrometry score is associated with urine leak after partial nephrectomy. BJU Int 2011;108:67-72. http://dx.doi.org/10.1111/i.1464-410X.2010.09837.x

5. Cha EK, $\mathrm{Ng} C K$, Jeun $B$, et al. Preoperative radiographic parameters predict long-term renal impairment following partial nephrectomy. World J Urol 2011. Epub 2011 May 21. http://dx.doi.org/10.1007/ s00345-011-0694-z

6. Hew MN, Baseskioglu B, Barwari K, et al. Critical Appraisal of the PADUA Classification and Assessment of the R.E.N.A.L. Nephrometry Score in Patients Undergoing Partial Nephrectomy. J Urol 201 1;186:82-6. http://dx.doi.org/10.1016/i.juro.2011.03.020

7. Simhan J, Smaldone MC, Tsai KJ, et al. Objective Measures of Renal Mass Anatomic Complexity Predict Rates of Major Complications Following Partial Nephrectomy. Eur Urol 2011;60:724-30. Epub 2011 May 25. http://dx.doi.org/10.1016/i.eururo.2011.05.030

8. Waldert $M$, Waalkes $S$, Klatte $T$, et al. External validation of the preoperative anatomical classification for prediction of complications related to nephron-sparing surgery. World J Urol 2010;28:531-5. http:// dx.doi.org/10.1007/s00345-010-0577-8

9. Okhunov Z, Rais-Bahrami S, George AK, et al. The Comparison of Three Renal Tumor Scoring Systems: C.Index, P.A.D.U.A., and R.E.N.A.L. Nephrometry Scores. J Endourol 2011;25:1921-4. Epub 2011 Sep 9.

10. Huang WC, Elkin EB, Levey $A S$, et al. Partial nephrectomy versus radical nephrectomy in patients with small renal tumors-is there a difference in mortality and cardiovascular outcomes? J Urol 2009; 181:5561; discussion 61-2.

11. Huang WC, Levey AS, Serio AM, et al. Chronic kidney disease after nephrectomy in patients with renal cortical tumours: a retrospective cohort study. Lancet Oncol 2006;7:735-40. http://dx.doi.org/10.1016/ S1470-2045(06)70803-8

12. Thompson RH, Boorijian SA, Lohse CM, et al. Radical nephrectomy for pTla renal masses may be associated with decreased overall survival compared with partial nephrectomy. J Urol 2008;179:468-71; discussion 472-3.

13. Clark AT, Breau RH, Morash C, et al. Preservation of renal function following partial or radical nephrectomy using 24-hour creatinine clearance. Eur Urol 2008;54:143-9. http://dx.doi.org/10.1016/i.euru10.2008.03.037

14. Lesage $\mathrm{K}$, Joniau $S$, Fransis $\mathrm{K}$, et al. Comparison between open partial and radical nephrectomy for renal tumours: perioperative outcome and health-related quality of life. Eur Urol 2007;51:614-20. http:// dx.doi.org/10.1016/i.eururo.2006.10.040

15. Van Poppel H, Da Pozzo L, Albrecht W, et al. A prospective, randomised EORTC intergroup phase 3 study comparing the oncologic outcome of elective nephron-sparing surgery and radical nephrectomy for low-stage renal cell carcinoma. Eur Urol 2011;59:543-52. http://dx.doi.org/10.1016/i.eururo.2010.12.013

Correspondence: Dr. Rodney H. Breau, Department of Surgery, Division of Urology, Ottawa University Hospital, General Campus, 501 Smyth Rd, Ottawa, ON, K1H 8L6; fax: 613-737-3019; rbreau@toh.on.co 